\title{
Strain Associated with Surface-Penetrating Dislocations Visible by Electron Channeling Contrast Imaging
}

\author{
Mincong Liu and Yoosuf N. Picard \\ Department of Materials Science and Engineering, Carnegie Mellon University, Pittsburgh, PA, 15213
}

Electron channeling contrast imaging (ECCI) is a non-destructive technique that can be used to image defects in crystalline materials. ECCI operates by selection of a certain diffraction condition, where a slight deviation from Bragg angle will induce a sharp change in the backscattered electron (BSE) yield. Defects such as dislocations can cause strong local deformation, or strain, in a crystal that will lead to characteristic channeling contrast profiles that correspond both to the acting diffraction condition and dislocation Burgers vector. Since strain is both a quantifiable measure of the deformation and the cause of the ECCI dislocation contrast, a question arises whether there is a way to relate the spatial size of contrast features to corresponding three-dimensional strain components. The magnitude of strain visible by ECCI has not been well determined and is the focus of this study.

For this work, ECCI is employed to analyze contrast features corresponding to specific dislocations in $\mathrm{SrTiO}_{3}$ (001) single crystals. ECCI is performed using an FEI Quanta 600 field emission gun (FEG) scanning electron microscope (SEM). The electrons are collected using a pole-piece-mounted annulus BSE detector. The accelerating voltage is kept at $20 \mathrm{kV}$ and beam current (nAs) is maintained for all analysis.

ECCI images taken at three different diffraction or channeling conditions (g) are shown in Figure 1. Figure 1(a), (c) and (e) are images of a Berkovich nanoindent in the $\mathrm{SrTiO}_{3}(001)$ substarte surface recorded at $\mathbf{g}=100,010$ and 110 diffraction conditions, respectively. The spot features near the indent denote surface penetrating dislocations. Figure 1(b), (d) and (f) are magnified views of two spot features as denoted in black rectangles in Figure 1(a), (c) and (e). These spot features exhibit strong bright contrast with weaker contrast forming a "tail," which corresponds to comet-like features previously observed in $\mathrm{SrTiO}_{3}$ (001) [1]. This type of contrast feature is indicative of surface-penetrating screw type dislocation with dislocation line inclined 45 degrees to the surface.

For this type of surface-penetrating screw dislocation, the elastic strain field near the dislocation line can be calculated using three-dimensional displacement solutions based on elastic theory [2]. Figure 2(a) shows the order of strain component $\varepsilon_{\mathrm{yy}}$ near dislocation line at a depth of $100 \mathrm{~nm}$, where the y-axis has been configured to be parallel to the [010] direction. The origin is chosen at the point where the dislocation line interests the horizontal plane. Figure 2(b) is the ECCI image of a single surfacepenetrating screw dislocation. Comparative spatial analysis of both ECCI contrast features and calculated elastic strain field indicates that $10^{-4}$ strain corresponds to the limit where channeling contrast is visible. These estimates indicate that magnitude strains in the $10^{-3}$ to $10^{-4}$ levels could be qualitatively imaged by ECCI. Cross-correlation electron backscatter diffraction (EBSD) is also sensitive to these levels of strain [3], so future coordinated ECCI and EBSD studies could be pursued.

References:

[1] R. J. Kamaladasa et al, Journal of Electronic Materials 40 (2011) 2222.

[2] S. J. Shaibani and P.M. Hazzledine, Philosophical Magazine A 44 (1981) 657.

[3] T.B. Britton et al, Ultramicroscopy 135 (2013) 126. 

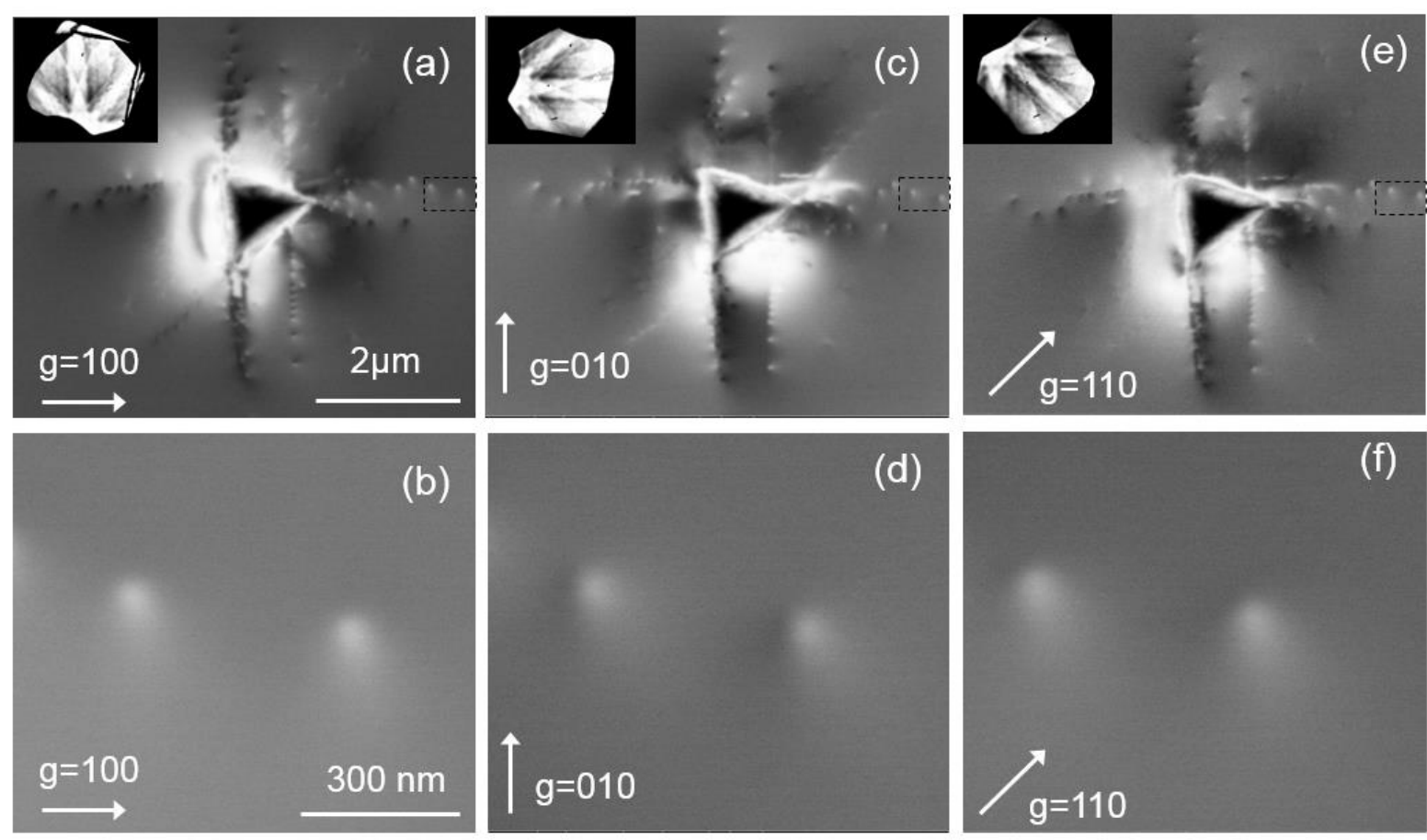

Figure 1. ECCI micrographs of a Berkovich indent in a $\mathrm{SrTiO}_{3}(001)$ single-crystal substrate surface at (a) $\mathbf{g}=100$, (c) $\mathbf{g}=010$ and (e) $\mathbf{g}=110$ diffraction conditions. Black dotted rectangles denote location of magnified ECCI analysis for two dislocations presented at (b) $\mathbf{g}=100$, (d) $\mathbf{g}=010$ and (f) $\mathbf{g}=110$ diffraction conditions.
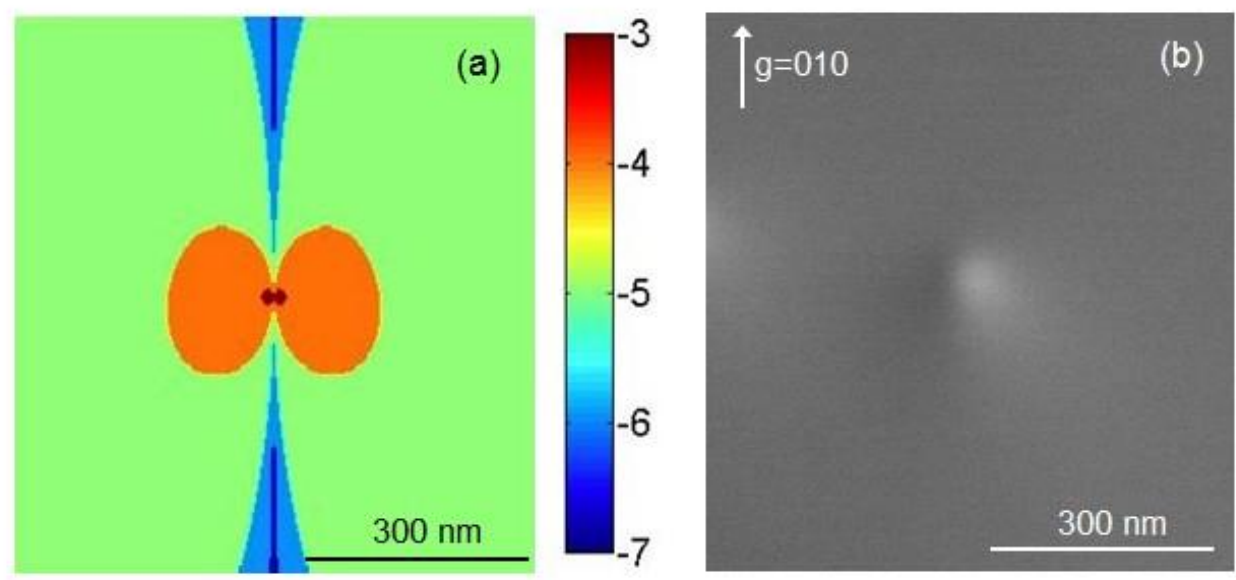

Figure 2. (a) Calculated order of magnitude strain component $\varepsilon_{\text {yy }}$ at a depth of $100 \mathrm{~nm}$ below the $\mathrm{SrTiO}_{3}$ (001) surface induced by a screw dislocation line. (b) ECCI image of corresponding surface-penetrating dislocation. 